\title{
Inhibition of lymphocyte function associated antigen 1 by LFA878 induces apoptosis in multiple myeloma cells and is associated with downregulation of the focal adhesion kinase/ phosphatidylinositol 3 kinase/Akt pathway
}

\author{
RALF SCHMIDMAIER, SONJA MANDL-WEBER, LEANDER GAUL, PHILIPP BAUMANN, \\ IRMGARD BUMEDER, CHRISTIAN STRAKA and BERTOLD EMMERICH \\ Department of Hematology and Oncology, Medizinische Klinik Innenstadt, \\ Klinikum der Universität München, Ziemssenstr. 1, D-80336 Munich, Germany
}

Received April 12, 2007; Accepted June 5, 2007

\begin{abstract}
Multiple myeloma (MM) is still an incurable disease and adhesion of MM cells to bone marrow stromal cells is one of the hallmarks of the disease. Lymphocyte function associated antigen 1 (LFA-1) is an adhesion molecule that mediates lymphocyte adhesion, but its role in MM is only poorly understood. The aim of the presented study was to improve knowledge on LFA-1 and associated pathways in MM for the development of molecular targeted therapies. We demonstrate that LFA-1 is expressed in U266, RPMI-8226, OPM-2, and NCI-H929 MM cell lines and in primary cells of eight tested patients. The LFA-1 inhibitor LFA878 induces apoptosis in all four cell lines as revealed by annexin V staining and caspase 3 cleavage. Apoptosis is not hampered by adhesion to stromal cells. Additionally, the soluble ligand, intracellular adhesion molecule 1 (ICAM-1), which is increased in the serum of MM patients, does not protect from melphalaninduced apoptosis. Western blots demonstrate downregulation of FAK, PI3-K, and Akt upon LFA878 treatment. Additionally, sequential inhibition of the pathway by simultaneous application of Src family kinase or PI3-K inhibitors significantly increases LFA878 induced apoptosis. We conclude that LFA-1/FAK/PI3-K/Akt is a survival pathway in MM and that targeted inhibition may provide new therapeutic options.
\end{abstract}

\section{Introduction}

Multiple myeloma (MM) is still an incurable hematological neoplasm that is characterized by homing, survival, and proliferation of malignant, antibody producing plasma cells in the bone marrow. All clinically relevant symptoms (cytopenia, hyperproteinemia and proteinuria with renal insufficiency,

Correspondence to: Dr Ralf Schmidmaier, Medizinische Klinik Innenstadt, Abteilung Hämatologie und Onkologie, Klinikum der Universität München, Ziemssenstr. 1, D-80336 München, Germany E-mail: ralf.schmidmaier@med.uni-muenchen.de

Key words: multiple myeloma, survival pathway, apoptosis, lymphocyte function associated antigen 1, LFA878 hypercalcemia, and osteolysis) are due to the infiltration of the whole bone marrow by MM cells, while other solid and lymphoid organs including the peripheral blood are usually spared. Plasma cell leukemia is rare and in most cases a sign of end stage disease. From these clinical observations and from many preclinical studies it is evident, that adhesion of MM cells to the bone marrow cells plays a crucial role in the pathophysiology of MM (1). Adhesion is mainly mediated by very late antigen 4 (VLA-4), lymphocyte function associated antigen 1 (LFA-1), and CD44 (2). Adhesion of MM cells leads to the secretion of stimulatory cytokines (2), upregulation of adhesion molecules $(3,4)$, proliferation of MM cells, and drug resistance (5). VLA-4 is strongly expressed on MM cells and targeting of VLA-4 provides a possible treatment strategy for MM (6). In line with these results we have previously shown that inhibition of LFA-1 by monoclonal antibodies or specific inhibitors reduces cell adhesion mediated drug resistance in MM (5). Intracellular adhesion molecule 1 (ICAM-1), the ligand of LFA-1, is expressed on bone marrow stromal cells and osteoblasts and LFA-1/ICAM-1 interaction is crucial for the pathophysiology of MM (7). Interestingly, LFA-1 positive MM cells injected into mice induce MM, whereas LFA-1 negative MM cells do not, although transendothelial migration and bone marrow homing capacities of MM cells are not affected by LFA-1 inhibition (8). So far, almost nothing is known about LFA-1 signalling pathways in MM. We therefore performed in vitro studies to improve knowledge on the role of LFA-1 in MM and to develop new therapeutic strategies for this incurable disease.

\section{Materials and methods}

Cells. U266, RPMI-8226, and HS-5 cell lines were obtained from the American Type Culture Collection (Rockville, MD), NCI-H929 and OPM-2 MM cells from the German Collection of Microorganisms and Cell Cultures (DSMZ, Braunschweig, Germany). Cells were grown in RPMI-1640 medium (Boehringer, Ingelheim, Germany) containing 10\% heat-inactivated fetal calf serum (FCS; Boehringer) in a humidified atmosphere $\left(37.5^{\circ} \mathrm{C} ; 5 \% \mathrm{CO}_{2}\right)$, and seeded at a concentration of $1 \times 10^{5}$ cells $/ \mathrm{ml}$. Coculture experiments to 
evaluate cell adhesion mediated drug resistance to LFA878 were performed as described before $(5,9)$. Briefly, a confluent layer of HS-5 was grown and then MM cells were added for several hours. After adhesion LFA878 was added. Cells were detached by pipetting vigorously and stained with CD138 and/or CD38. MM cells were identified by three colour flow cytometry and percentage of propidium iodide positive cells was determined. Primary MM cells were obtained from consecutive patients after informed consent was obtained for the use of residual bone marrow material after routine diagnostics. Serum was collected from consecutive patients in the multiple myeloma outpatient clinic after informed consent was obtained. The study was approved by the ethics committee of the University of Munich.

Reagents. Annexin V-FITC was purchased from Caltag Laboratories (Hamburg, Germany), propidium iodide and PP2 from Calbiochem (Schwalbach, Germany), rhICAM-1 from R\&D Systems (Wiesbaden-Nordenstadt, Germany). WST-1 was purchased from Roche (Penzberg, Germany), melphalan from Sigma-Aldrich (Taufkirchen, Germany), LFM-A13, Herbimycin A, PP1, and LY294002 from Biomol (Hamburg, Germany). CD18-FITC and CD11a-FITC were purchased from Immunotech (Marseille, France), CD45-FITC from BD Biosciences (Heidelberg, Germany). Primary Western blot antibodies (actin, cyclin A, cyclin B1, cyclin D1, p21, p53, Rb, 14-3-3 o, PI3-K, ERK-2, ERK-1, JAK2, STAT3, c-IAP 1, c-IAP 2, Bcl-2, Bad, Bim, Bax) were purchased from Santa Cruz Biotechnology, Inc. (Heidelberg, Germany) or (cleaved Caspase-3, Src, SHP-2, FAK, Paxillin, Csk, Akt, MEK1/2, FLIP, XIAP, Cdc25C) from Cell Signaling Technology, Inc. (Frankfurt am Main, Germany) or (PTP-1B, anti-p130 CAS) from Upstate (Biomol, Hamburg, Germany) or (PTP $\alpha$ ) from Abcam (Biozol, Eching, Germany). Anti-rabbit and anti-mouse immunoglobulin $\mathrm{G}$ horseradish peroxidase-conjugated antibodies were obtained from Amersham Biosciences (Uppsala, Sweden) and peroxidase-conjugated anti-goat immunoglobulins from Dako (Glostrup, Denmark). LFA878 was kindly provided by Novartis (Basel, Switzerland).

Analysis of cell death and apoptosis by flow cytometry. Cells were stained with fluorescein isothiocyanate-conjugated annexin $\mathrm{V}$ and propidium iodide (PI). Briefly, after two washes with washing buffer $\left(8 \mathrm{~g} \mathrm{NaCl}, 0.2 \mathrm{~g} \mathrm{KCl}, 1.44 \mathrm{~g} \mathrm{Na}_{2} \mathrm{HPO}_{4}\right.$, $0.24 \mathrm{~g} \mathrm{KH}_{2} \mathrm{PO}_{4}, 11 \mathrm{H}_{2} \mathrm{O}, \mathrm{pH}$ 7.2), cells were resuspended in binding buffer (10 mM HEPES/NaOH, pH 7.4, $140 \mathrm{mM}$ $\mathrm{NaCl}, 2.5 \mathrm{mM} \mathrm{CaCl}_{2}$ ). A total of $100 \mu 1$ of this cell suspension was incubated with $5 \mu \mathrm{l}$ annexin V-FITC and $10 \mu \mathrm{l}$ of $50 \mu \mathrm{g} / \mathrm{ml}$ PI for $15 \mathrm{~min}$ at room temperature in the dark. Cells were analyzed by flow cytometry (Coulter Epics XL-MCL; System II) within $30 \mathrm{~min}$.

Analysis of cell cycle. Cells were fixed overnight with $70 \%$ $(\mathrm{w} / \mathrm{v})$ ice-cold ethanol. After two washes with ice-cold phosphate-buffered saline (PBS), the fixed cells were resuspended in $1 \mathrm{ml}$ of PBS containing $40 \mu \mathrm{g} / \mathrm{ml}$ propidium iodide (PI) and $500 \mathrm{U} / \mathrm{ml}$ RNase A. Following incubation for $30 \mathrm{~min}$ in the dark at room temperature, the cells were analyzed on a Coulter Epics XL-MCL flow cytometer (Beckman Coulter,
Krefeld, Germany) using the System II software. To identify apoptotic populations, objects with fluorescence values $<20 \%$ of the G1 peak were gated out. As previously described, the PI fluorescence signal peak versus the integral was used to discriminate $\mathrm{G} 2 / \mathrm{M}$ cells from $\mathrm{G} 0 / \mathrm{G} 1$ doublets.

Proliferation assay. For quantification of the cells in suspension the WST-1 viability assay protocol was used as recommended by the manufacturer (Roche, Penzberg, Germany). Absorbance at $440 \mathrm{~nm}$ was measured using a microplate enzyme-linked immunosorbent assay reader to detect metabolically intact cells (reference wavelength: $680 \mathrm{~nm}$ ).

Surface expression of antigens. For analysis of cell surface markers $10^{6}$ cells were washed with PBS and resuspended in $1 \mathrm{ml}$ PBS with $10 \%$ FCS. Aliquots of $100 \mu 1$ of cells were incubated at $4{ }^{\circ} \mathrm{C}$ with fluorochrome conjugated antibodies for $10 \mathrm{~min}$ in the dark. After centrifugation the cells were resuspended in $500 \mu 1$ of PBS with $10 \%$ FCS and flow cytometry was performed.

Enzyme-linked immunosorbent assay (ELISA). Samples were centrifuged and serum was frozen in aliquots at $-20^{\circ} \mathrm{C}$. Aliquots were defrosted, a standard curve of cytokines was established, and serum levels of ICAM-1 were calculated ( $\mathrm{pg} / \mathrm{ml})$ by using the quantikine ${ }^{\circledR}$ high sensitivity enzyme linked immunosorbent assay (ELISA) kit from R\&D systems, Wiesbaden, Germany. Means of duplicate samples were used for calculation of the serum levels. The procedure was performed as recommended by the manufacturer.

Immunoblot analysis. Cells were washed three times in ice-cold PBS and lysed in a buffer containing $10 \mathrm{mM}$ Tris- $\mathrm{HCl}(\mathrm{pH} 7.6)$, $137 \mathrm{mM} \mathrm{NaCl}, 1 \mathrm{mM} \mathrm{Na} \mathrm{VO}_{4}, 10 \mathrm{mM} \mathrm{NaF}, 10 \mathrm{mM}$ EDTA, $1 \%(\mathrm{v} / \mathrm{v}) \mathrm{NP}-40$ with the addition of $10 \mu \mathrm{g} / \mathrm{ml}$ aprotinin, $10 \mu \mathrm{g} / \mathrm{ml}$ leupeptin, and $1 \mathrm{mM}$ phenylmethylsulfonylfluoride (PMSF). Protein concentration was adjusted using a colorimetric assay (Bio-Rad Protein Assay, Bio-Rad, Munich, Germany). Proteins were subjected to SDS-polyacrylamide gel electrophoresis and transferred to a polyvinylidene difluoride (PVDF) membrane (Millipore, Germany). The transfer buffer contained $25 \mathrm{mM}$ Tris-HCl, $192 \mathrm{mM}$ glycine, 0.037 (w/v) SDS, and $20 \%(\mathrm{v} / \mathrm{v})$ methanol. The membranes were blocked with TBS containing $5 \%$ dried milk and $0.05 \%$ Tween-20 (TBS/Tween). After washing four times with TBS/Tween, the membranes were incubated with appropriate primary antibodies and visualized by autoradiography using the ECL Western blot detection system (Amersham Pharmacia, Freiburg, Germany).

Statistical analyses. Mean values with standard deviations from representative experiments are shown in the figures. The Kruskal-Wallis one-way analysis of variance on ranks was used to determine the statistical significance of treatment results. $\mathrm{P}<0.05$ was considered statistically significant.

\section{Results}

LFA-1 expression on multiple myeloma cells and SICAM-1 serum levels in MM patients. The starting point of our study 


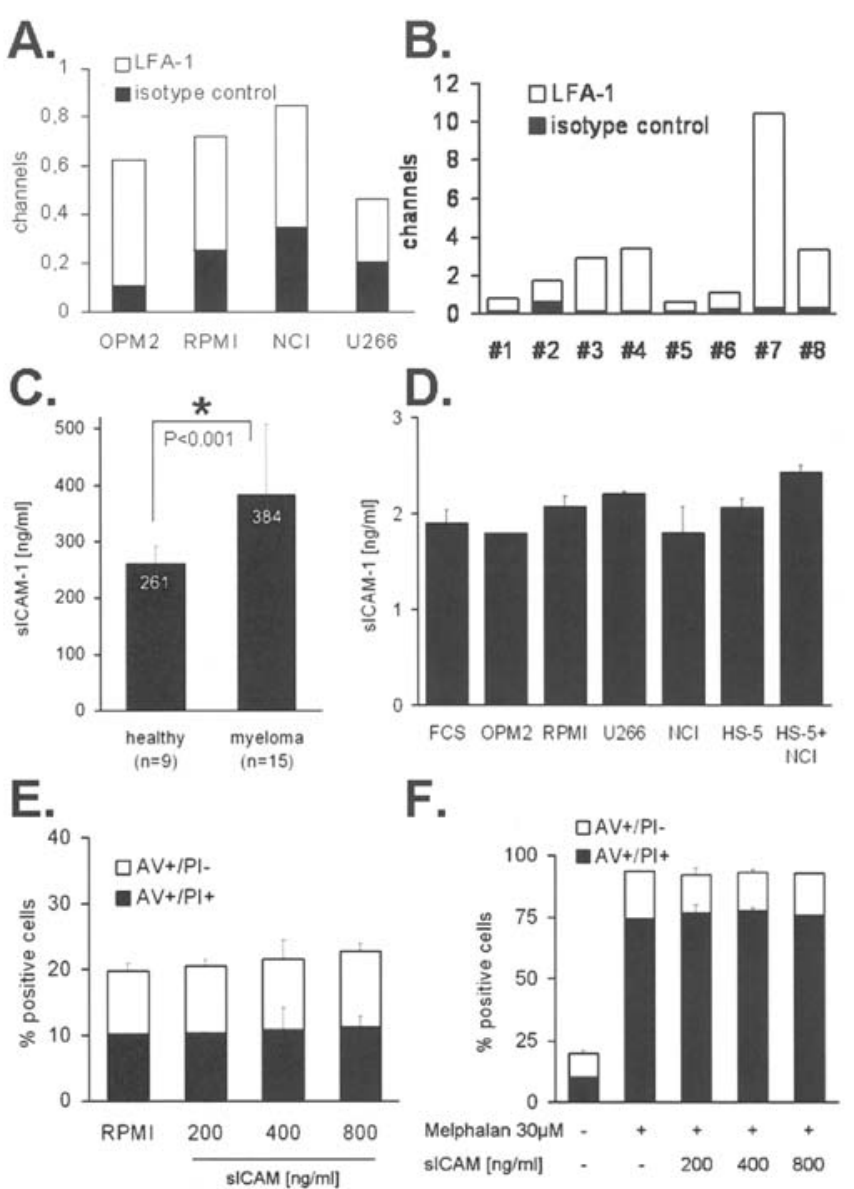

Figure 1. LFA-1 expression on multiple myeloma cells and sICAM-1 serum levels in MM patients. A and B, Expression of LFA-1 (CD11a) integrin was determined by flow cytometry in four (A) MM cell lines (OPM-2, RPMI-8226, NCI-H929, U266) and primary MM cells from bone marrow of eight consecutive patients (B). CD11a was FITC conjugated and median fluorescence in channels is shown in comparison to unspecific fluorescence of polyclonal antibodies of the same isotype. C and D, Soluble ICAM-1 (sICAM-1) was determined by ELISA in the serum of 9 healthy volunteers and of $15 \mathrm{MM}$ patients (C), in the supernatant of MM (OPM-2, RPMI-8226, U266, NCI-H929) and bone marrow stromal cell lines (HS-5), and in fetal calf serum (FCS) (D). Asterisk indicates statistically significant results $(\mathrm{P}<0.05)$. E, Recombinant human ICAM-1 (sICAM-1) was added at the indicated concentrations for $48 \mathrm{~h}$ to RPMI-8226 MM cells and apoptosis was determined by flow cytometry after staining with propidium iodide (PI) and annexin V (AV). F, Cell death was induced by $30 \mu \mathrm{M}$ melphalan and influence of sICAM-1 on cell death and apoptosis was determined.

was to confirm that LFA-1 is expressed on MM cells. In all four tested MM cell lines we found a quite constant expression of CD11a (Fig. 1A). Additionally, in all eight consecutive patient samples primary MM cells expressed LFA-1 (Fig. 1B). The difference between some patients was striking, but most samples were quite similar. ICAM-1 is a ligand of LFA-1 and is expressed on bone marrow stromal cells. Furthermore, the soluble form of ICAM-1 (sICAM-1) is detectable in the serum. Addressing the importance of ICAM-1/LFA-1 interaction in MM we analysed sICAM-1 serum levels by ELISA in $15 \mathrm{MM}$ patients and nine healthy volunteers. Thus revealed a statistically significant difference $(\mathrm{P}<0.001)$ with mean values of $261 \mathrm{ng} / \mathrm{ml}$ and $384 \mathrm{ng} / \mathrm{ml}$, respectively (Fig. 1C). Furthermore, we determined sICAM-1 in the supernatant of MM (OPM-2, RPMI-8226, U266, NCI-H929) and bone marrow stromal cell lines (HS-5), and in fetal calf serum (FCS). In comparison to the measured serum levels cell line supernatants are almost free of sICAM-1 (Fig. 1D). In particular, in the supernatant of coincubated MM and stromal cells sICAM-1 levels were not elevated. Even in fetal calf serum levels were very low, although this could be due to the human specificity of the antibody. However, considering these results we suggested that addition of sICAM may influence apoptosis of MM cells. Therefore, keeping in mind the mean serum level of $384 \mathrm{ng} / \mathrm{ml}$, RPMI-8226 MM cells were treated with 200,400 , and $800 \mathrm{ng} / \mathrm{ml} \mathrm{rhICAM}-1$ and the percentage of annexin $\mathrm{V} /$ propidium iodide positive cells was determined by flow cytometry. Spontaneous apoptosis of RPMI-8226 was not altered by SICAM-1 (Fig. 1E). Furthermore, RPMI-8226 cells were treated with melphalan and rhICAM-1 was added in increasing concentrations. Melphalan-induced apoptosis of MM cells was not reduced by sICAM-1 (Fig. 1F). Similar results were obtained using NCI-H929 MM cells.

Inhibition of LFA-1 by the specific inhibitor LFA878 induces apoptosis in multiple myeloma cells. Since LFA-1 inhibitors become available for clinical use in lymphocytic diseases such as allergy (10), psoriasis (11), or organ rejection (12), it seemed interesting to investigate the effects of LFA-1 inhibitors on MM cells. We chose the statin derivative LFA878, which binds to an allosteric site of the alphaL I domain termed the lovastatin site (L-site), and which lacks hydroxymethylglutaryl coenzyme A (HMG-CoA) reductase activity (13). Previously, we and others have shown significant anti-MM activity of statins $(5,14)$. First, we incubated the four MM cell lines with LFA878 in a dose-dependent manner and determined signs of apoptosis [annexin V (AV) positive] and cell death [propidium iodide (PI) positive] by flow cytometry. Fig. 2A shows dose-dependent induction of apoptosis by LFA878 in all tested cell lines. As we observed a rapid increase of apoptosis/cell death in most cell lines, we narrowed the used concentrations and observed strict dose-dependence in these experiments (Fig. 2A). Additionally, we wanted to prove that LFA878 mediated cell death is really due to apoptosis. Fig. 2B shows dose-dependent cleavage of caspase 3, the central effector caspase of the apoptotic cascade. The concentrations necessary to induce apoptosis and cell death are quite similar to concentrations needed with potent anti-myeloma cytotoxic drugs such as melphalan (15). However, we questioned whether LFA878-induced apoptosis is disease specific or whether healthy leukocytes are comparably sensitive to this drug. Peripheral blood mononuclear cells (PBMC) remained quite unaffected by the treatment with LFA878, suggesting a clear disease-specific effect (Fig. 2C). Only $9 \%$ of cells become annexin $\mathrm{V}$ and propidium iodide positive at $100 \mu \mathrm{M}$, whereas all MM cells are dead at this concentration. Additionally, we performed a time course experiment with NCI-H929 MM cells with different LFA878 concentrations. Fig. 2D shows that the substantial induction of irreversible apoptosis requires about one day. Beyond $24 \mathrm{~h}$ there is no further increase of cell death and apoptosis. With respect to the pathophysiology of MM cells in the microenvironment of bone marrow stromal cells NCI-H929 MM cells were grown in contact with HS5 bone marrow 
A.
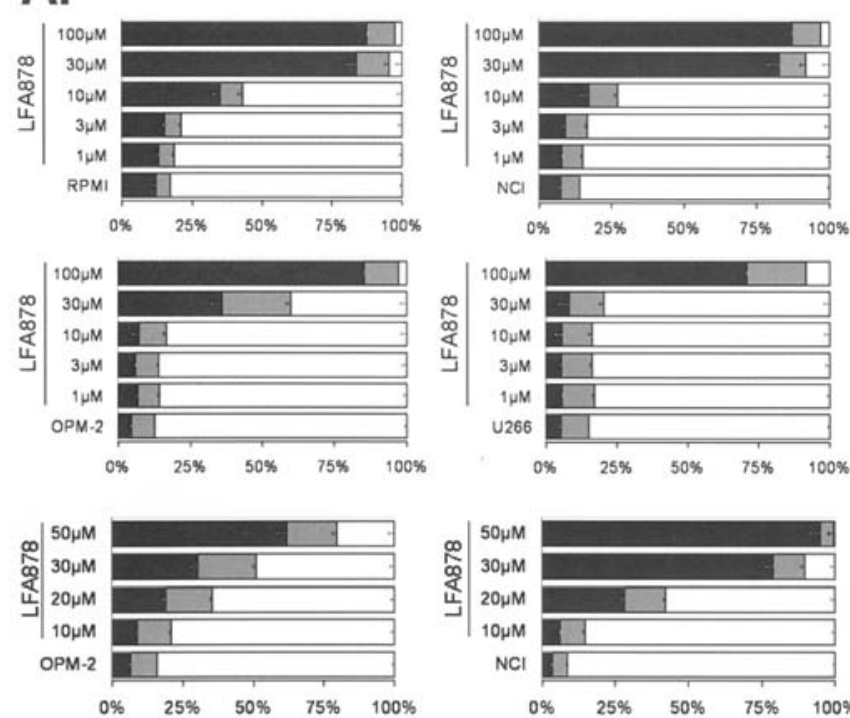

$0 \% \quad 25 \% \quad 50 \% \quad 75 \% \quad 100 \%$

late apoptosis (PI+/AV+)

early apoptosis (PI-/AV+)

viable cells (PI-/AV-)

B.

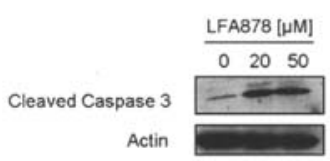

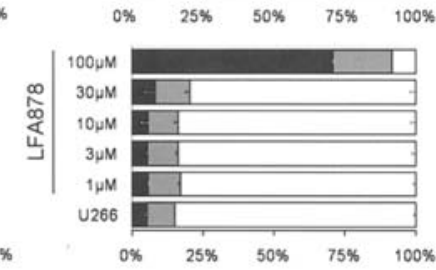

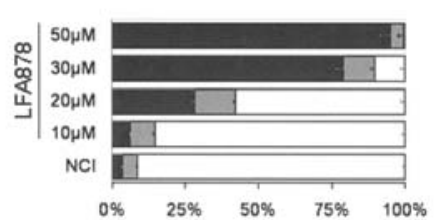

C.

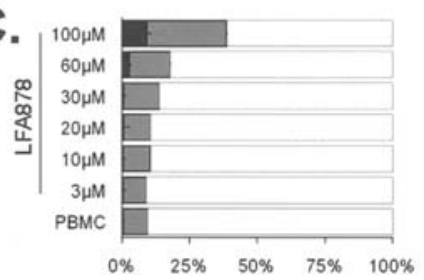

D.

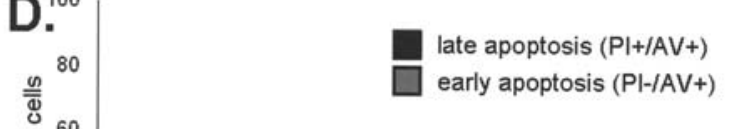

$1 \mathrm{~h}$

$3 \mathrm{~h}$

$6 \mathrm{~h}$

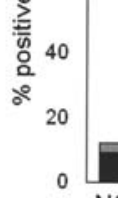

$\mathrm{NCl}$

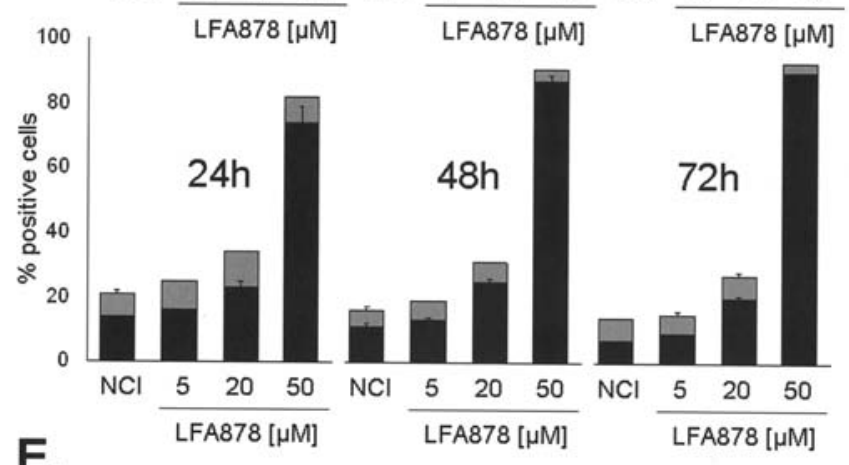

E.

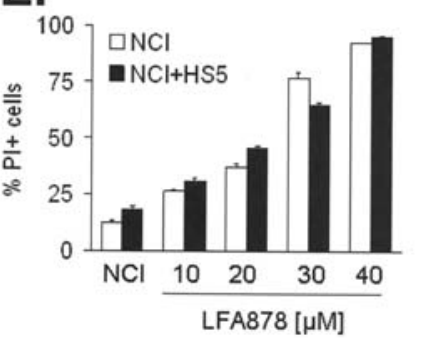

Figure 2. Inhibition of LFA-1 by the specific inhibitor LFA878 induces apoptosis in multiple myeloma cells. A, MM cells (RPMI-8226, NCI-H929, OPM-2, U266) were treated for $48 \mathrm{~h}$ with increasing concentrations of LFA878 as indicated in the figure. Cell death and apoptosis were determined by flow cytometry after staining with annexin V-FITC (AV) and propidium iodide (PI). B, OPM-2 MM cells were incubated with LFA878 at 20 and $50 \mu$ M for 48 h. Cell lysates were directly subjected to SDS-PAGE, transferred to membranes and blotted with anti cleaved caspase 3 antibodies. Actin shows equal loading. C, Peripheral blood mononuclear cells (PBMC) were incubated for $48 \mathrm{~h}$ with increasing concentrations of LFA878 as indicated in the figure. Annexin V (AV) and propidium iodide (PI) expression was determined by flow cytometry to detect signs of apoptosis and/or cell death. D, NCI-H929 MM cells were treated with increasing concentrations of LFA878 for 1, 3, 6, 24, 48, and $72 \mathrm{~h}$. Propidium iodide (PI) and annexin V (AV) positive cells were determined by flow cytometry. E, NCI-H929 MM cells were treated with increasing concentrations of LFA878 as indicated in the figure in the presence or absence of HS-5 bone marrow stromal cells. MM cells were identified by CD38 and CD138 staining and cell death was determined by propidium iodide (PI) uptake.

stromal cells and cell death was determined after addition of LFA878. Fig. 2E demonstrates that LFA878 induced cell death remains unaffected by the presence of bone marrow stromal cells. Since former studies have shown that LFA-1 and ICAM-1 mediate homotypic MM cell aggregation (16), we systematically controlled this by microscopy, but could neither observe development of aggregations, nor release of aggregates upon LFA878 treatment. Additionally, LFA878 treated MM cells were incubated at increasing MM cell concentrations to narrow the cells and herewith to promote cell cell contact. No statistically significant differences could be observed (data not shown).

Intracellular effects of LFA878 mediated LFA-1 inhibition. First, we addressed the influence of LFA-1 inhibition on proteins that regulate the cell cycle. It is well known that most cytotoxic drugs lead to alterations of cell cycle progression. This is mainly due to regulatory cell cycle arrest via checkpoint mechanisms to enable repair of the cells (for comprehensive review see ref. 17). Indeed, Fig. $3 \mathrm{~A}$ demonstrates that
LFA878 treatment leads to accumulation of cells in the G1/G0 phase (Fig. 3A). At the G1/S checkpoint cell cycle arrest is p53-dependent and Fig. 3B shows that p53 was increased upon LFA878 treatment, whereas p21 remained unaltered. In addition 14-3-3 $\sigma$, a protein that regulates the intracellular trafficking of different proteins including the CDK activating kinase cdc25c, was strongly upregulated upon LFA878. 14-3-3 $\sigma$ exports cdc25 and cyclin B from the nucleus, which was also the case in our MM model (Fig. 3B). Cyclin B1 was slightly decreased, whereas no changes were observed regarding cyclin B1, cyclin D1, and CDK1. We did not observe a significant or relevant inhibition of proliferation by LFA878 (Fig. 3C). Of note, the obvious decrease of viable RPMI-8226 cells is mainly due to apoptosis (compare with Fig. 2A). Despite the signs of cell cycle arrest we concluded that LFA-1 inhibition rather blocks a survival pathway than a proliferation pathway. Therefore, we determined intracellular expression levels of pro- and anti-apoptotic proteins upon LFA878 treatment by Western blotting. Bax, Bim, Bad, XIAP, cIAP-1, and cIAP-2 
A.

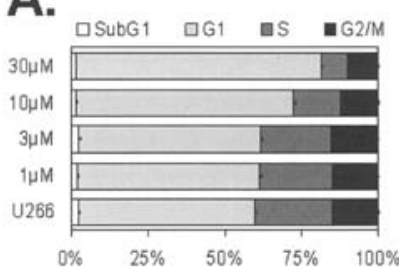

B.

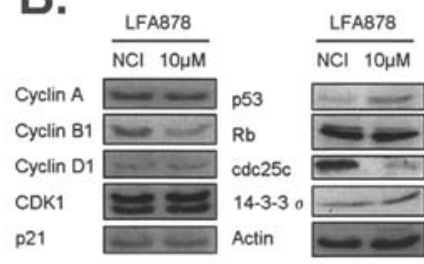

C.
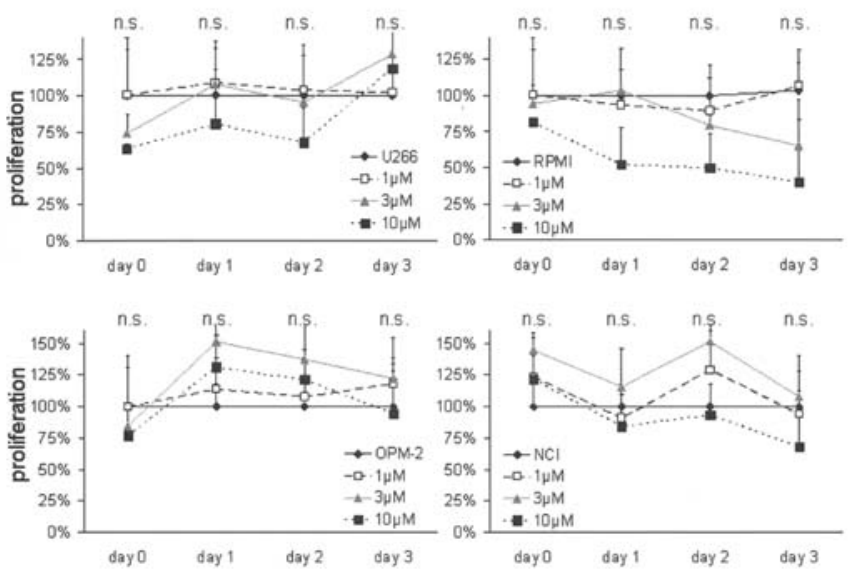
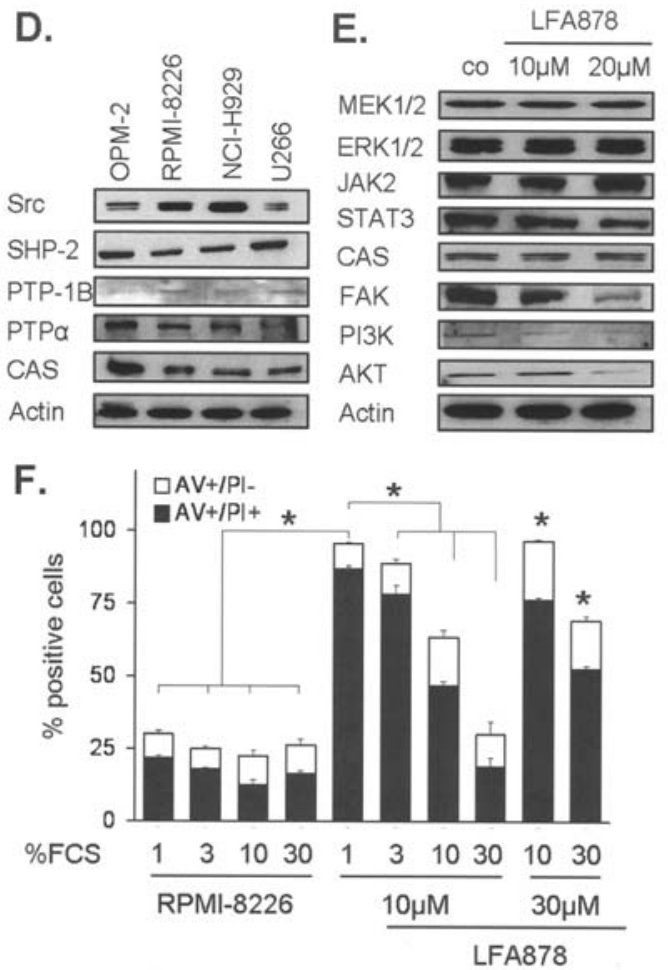

Figure 3. Intracellular effects of LFA878 mediated LFA-1 inhibition. A, U266 MM cells were incubated with LFA878 as indicated in the figure. Cell cycle analysis was performed with fixed cells after PI staining and several washings. B, NCI-H929 MM cells were incubated with LFA878 as indicated in the figure. Cell lysates were directly subjected to SDS-PAGE, transferred to membranes and blotted with specific antibodies as indicated. Actin shows equal loading. C, U266, RPMI-8226, OPM-2, and NCI-H929 MM cells were incubated with increasing concentrations as indicated in the figure. At days 0, 1, 2, and 3 the amount of viable cells was determined by WST-1 assay. Proliferation of untreated MM cells was set as $100 \%$ to allow comparison between concentrations and cell lines. D, OPM-2, RPMI-8226, NCI-H929, and U266 MM cells were lysed and directly subjected to SDS-PAGE, transferred to membranes and blotted with specific antibodies as indicated in the figure. Actin shows equal loading. The experiment was repeated three times with similar results. E, OPM-2 MM cells were treated with 10 and $20 \mu \mathrm{M}$ LFA878, cell lysates were directly subjected to SDS-PAGE, transferred to membranes and blotted with specific antibodies as indicated in the figure. Actin shows equal loading. The experiment was repeated three times with similar results. F, RPMI-8226 MM cells were treated with 10 or $30 \mu \mathrm{M}$ LFA878 for $48 \mathrm{~h}$ and increasing concentrations of fetal calf serum (FCS) as indicated in the figure. Apoptosis/cell death was determined by annexin $\mathrm{V}(\mathrm{AV})$ and propidium iodide $(\mathrm{PI})$. Asterisk indicates statistically significant results $(\mathrm{P}<0.05)$.

remained unaltered. LFA878 induced a slight increase of Bcl-2 and cFLIP, most likely reflecting a cellular stress response (data not shown). Next, we investigated signalling molecules immediately downstream of LFA-1. It is known that focal adhesion kinase (FAK) is expressed in MM cells, but we found no sufficient information regarding Src, SHP-2, PTP-1B, PTP $\alpha$, CAS in the literature. Therefore, we screened our four MM cell lines and found substantial expression of all these proteins with exception of PTP-1B, which was only slightly expressed (Fig. 3D). Upon LFA878 induced LFA-1 inhibition we found significant downregulation of FAK, PI3-K, and Akt (Fig. 3E), whereas CAS, MEK/ERK, and JAK2/STAT3 remained unaffected. Also Src, SHP-2, and PTP. expression levels were not changed. The experiments were repeated at least three times with similar results. We concluded that LFA-1 and the FAK/PI3-K/Akt pathway are constitutively active, although we were not able to stimulate this pathway by specific ligation with soluble or cell bound ICAM-1 (Fig. 1E and F and Fig. 2E). We hypothesized that a so far unknown soluble factor must be present in the medium/fetal calf serum (FCS). Therefore, we blocked the pathway by LFA878 and systematically increased the concentration of FCS in the culture medium. Indeed, LFA878induced apoptosis could be reversed by FCS (Fig. 3F). In addition, FAK downregulation was inhibited (data not shown).
LFA-1 inhibition in combination with downstream Src family kinase/PI-3 kinase inhibition. LFA-1 activates the Src/FAK complex and we have shown that Src is constitutively expressed in MM cells and that FAK, but not Src, is downregulated upon LFA878 treatment. We hypothesized that sequential pathway inhibition in terms of LFA-1 inhibition plus Src inhibition may synergistically induce apoptosis/cell death. Since not only Src but other Src family members may be involved we chose different inhibitors. PP1 inhibits Src, Lck, and Fyn. PP2 inhibits Lck, Fyn, and Hck. Herbimycin A inhibits Src, Yes, and other oncogene protein kinases. LFM-A13 is an inhibitor of the bruton's tyrosine kinase (BTK) which plays a crucial role in B cell function. BTK is activated by a two-step mechanism involving PI3-K and Lyn (18). LFM-A13, Herbimycin A, PP1, PP2 (50 $\mu \mathrm{M}, 1 \mu \mathrm{M}$, 10 and $50 \mu \mathrm{M}$ respectively) were combined with non-toxic concentrations of LFA878 $(10 \mu \mathrm{M})$ in OPM-2 MM cells. Fig. 4A shows that all four Src family kinase inhibitors act synergistically with LFA878 in terms of induction of apoptosis and furthermore, that Herbimycin A and PP2 themselves strongly induce apoptosis. Additionally, we tested the combination of LFA878 $(20 \mu \mathrm{M})$ with the PI3-K inhibitor LY294002 (Fig. 4B). Again, the combination of almost nontoxic LFA878 with non-toxic LY294002 resulted in significant apoptosis. 
A.

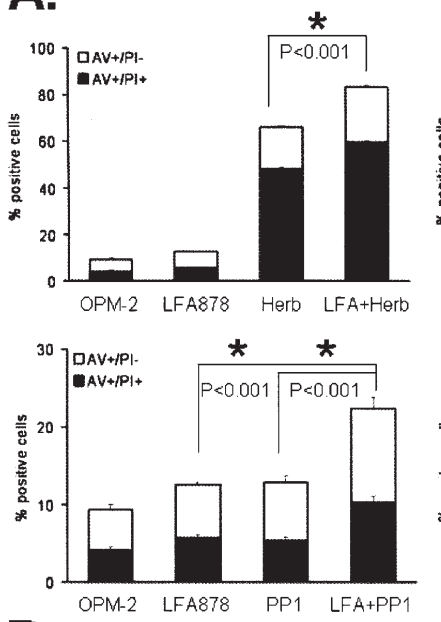

B.

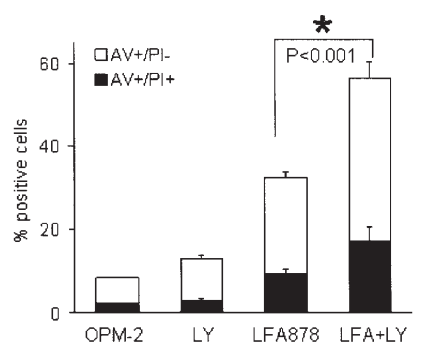

Figure 4. LFA-1 inhibition in combination with downstream Src family kinase/PI-3 kinase inhibition. A, OPM-2 MM cells (OPM2) were treated with Src family kinase inhibitors [1 $\mu \mathrm{M}$ Herbimycin A (Her), $50 \mu \mathrm{M}$ LFM-A13 (LFM), $10 \mu \mathrm{M}$ PP1, $50 \mu \mathrm{M}$ PP2] in the presence or absence of $10 \mu \mathrm{M}$ LFA878 (LFA). Cell death/apoptosis was determined by flow cytometry after annexin V (AV)/propidium iodide (PI) staining. Asterisk indicates statistically significant results $(\mathrm{P}<0.05)$. B, OPM-2 MM cells were treated for $48 \mathrm{~h}$ with $20 \mu \mathrm{M}$ LFA878 in combination with LY294002 (LY) as indicated in the figure. Apoptosis was determined by flow cytometry. Asterisk indicates statistically significant results $(\mathrm{P}<0.05)$.

\section{Discussion}

We demonstrate that LFA-1 is expressed in MM cells, cell lines and primary cells. Expression on cell lines is comparably constant, whereas quantitative expression on primary cells differs significantly. We have previously shown (3) that LFA-1 expression may be a negative response predictor and therefore targeting of the LFA-1 pathway could mean targeting aggressive and resistant MM. This is in accordance with two other studies that suggest higher expression of LFA-1 in aggressive MM $(19,20)$. Since it is well known that adhesion mediates proliferation of MM cells it is plausible that the LFA-1 expressing cells represent the proliferating compartment in this disease (21). In comparison to isotype control LFA-1 expression on primary MM cells is stronger than in cell lines. This may reflect the less adhesive growth of cell lines. Furthermore, we have shown that sICAM-1, the soluble ligand of LFA-1, is increased in the serum of MM patients in comparison to healthy controls. Similar investigations showed correlation of sICAM-1 with the stage of disease (22) and increase of sICAM-1 concomitant with thalidomide treatment, although it was without predictive value (23). However, the addition of SICAM-1 to the cell culture in our study did not influence survival or chemosensitivity. We increased the doses of sICAM-1 above levels measured in the bone marrow of MM patients $(500 \mathrm{ng} / \mathrm{ml})$ (24) up to $800 \mathrm{ng} / \mathrm{ml}$, but no MM-protective effect could be observed. We conclude that the increase of sICAM-1 in the serum of MM patients most likely reflects tumour burden but is not closely related to the primary pathophysiology of the disease.

Second, we tested whether targeting of LFA-1 by small molecule inhibitors provides a therapeutic option in MM. We demonstrate that LFA878, which is a lovastatin derivative and herewith an optimised LFA-1 inhibitor with strong antiinflammatory properties (13), strongly induces cell death in all tested MM cell lines accompanied by cellular events of apoptosis such as caspase 3 cleavage and loss of asymmetry of the cell membrane. Peripheral blood mononuclear cells do not become apoptotic upon LFA878 suggesting a diseasespecific effect. Additionally, our experiments revealed that in contrast to most other anti-MM agents (5) LFA878 induced apoptosis is not hampered by adhesion of MM cells to bone marrow stromal cells. Similar results have been obtained previously with simvastatin (14), which also has LFA-1 inhibitory activities. Although anti-LFA-1 strategies are increasingly in use in inflammatory and auto-immune diseases, to our knowledge this is the first study that demonstrates induction of apoptosis by an LFA-1 inhibitor in malignant disease and especially in MM. BIRT377 is a small molecule inhibitor of LFA-1 that showed antiproliferative effects in vitro, but failed to induce apoptosis (8). The particularity of LFA-1 in MM is reflected by the observation that inhibitory antibodies or potent small molecule inhibitors of VLA-4, which is much more strongly expressed than LFA-1, does not induce apoptosis in MM cells (Schmidmaier et al, unpublished data). However, modulation of LFA-1 expression by activation or inhibition of protein kinase $\mathrm{C}$ isoforms did not influence the chemosensitivity of the cells (Schmidmaier et al, unpublished data). Finally, since LFA-1 plays a central role in the immune function it is important to know that systemic LFA-1 inhibition is feasible and well tolerated in humans as reflected by the studies with efalizumab in psoriasis (25).

As only very little is known about LFA-1 signalling in MM we were interested in the intracellular mechanisms of LFA-1 inhibition. As it was previously described (8) we investigated the influence of LFA878 on cell cycle distribution and proliferation. Although we observed a G1 arrest upon LFA-1 inhibition no significant changes in the proliferative capacity could be observed. Considering the upregulation of p53 and the downregulation of cdc25c and 14-3-3 $\sigma$ we conclude that this represents cell cycle arrest upon cellular stress, namely the inhibition of survival pathways by LFA878. Typically p53 induction is associated with an increase of $\mathrm{p} 21$, an inhibitor of the cyclin B/cyclin dependent kinase (CDK) 1 complex. This was not observed upon LFA878 treatment. The analysis of pro- and antiapoptotic proteins of the bcl-2 and the IAP family did not reveal an explanation for the reduced survival upon LFA878. In addition, the expression of the signalling proteins of the main MM pathways, (MEK/ERK and JAK2/STAT3; for current review see ref. 26) remains unchanged, whereas PI3-K and Akt are 
significantly downregulated upon LFA-1 inhibition. Although there are no published data available regarding $\mathrm{B}$ cells or even plasma cells, similar LFA-1 induced activation of the PI3-K/Akt-pathways has been observed in T cells (27). We conclude this pathway is constitutively active in MM cells. This finding is of importance knowing that over-expression of IGF-1R is associated with $\mathrm{t}(4 ; 14)$, lack of CD45 expression, and herewith poor prognosis (28). Due to the lack of CD45 combined with a high level of IGF-1R expression, Akt signalling is of central importance in these patients. Also from a $\mathrm{T}$ cell model it is known that LFA-1 ligation may activate focal adhesion kinase FAK (29). FAK co-localizes with integrins and is herewith activated. Insulin-like growth factor-1 (IGF-1) treatment of MM cells is associated with FAK activation and IGF-1 induced adhesion of MM cells is associated with PI3-K/Akt pathway activation (30), whereas $\mathrm{MM}$ cell migration is associated with the PI3-K/PKCmicro and the PI3-K/RhoA pathway (31). In accordance with these results we can show that FAK is downregulated upon LFA878 mediated LFA-1 inhibition. We suggest FAK/PI3K/Akt downregulation as primary mechanism of LFA878 induced apoptosis in MM cells. As integrins do not possess intrinsic catalytic activity, the adhesion signals are mediated by activation of integrin-associated non-receptor protein tyrosine kinases such as FAK. Integrin-activated FAK binds to $\mathrm{Src}$, a non-receptor tyrosine kinase that was initially found as the transforming element of the Rous sarcoma virus ( $\mathrm{v}$ Src) (32). Many other tyrosine kinases have been identified and characterized (e.g. Src, Yes, Fgr, Yrk, Fyn, Lyn, Hck, Lck, and Blk). The disease-specific expression in human and the signalling pathways are still poorly understood. We demonstrate in the current work that Src is significantly expressed in all tested MM cell lines. Two of the main phosphorylation targets of the FAK-Src complex are the adaptor proteins paxillin and CAS (33). CAS is strongly expressed in our cell lines whereas we are not able to detect paxillin. We therefore suggest CAS to be involved in LFA-1 survival signalling. FAK-Src activity is additionally regulated by SHP-2, PTP-1B, PTP $\alpha$, and Csk in other cell models, but in our MM cell lines only SHP-2, PTP-1B, and PTP $\alpha$ could be detected. These CD45 associated proteintyrosine phosphatases (PTP) are permissive regulators of interleukin 6 dependent STAT3 and ERK1/2 mediated MM cell proliferation $(34,35)$ by a Src-dependent mechanism. The involvement of Src family kinases in MM survival pathways is further supported by our finding that PP2 and Herbimycin A intrinsically induce apoptosis in MM cells. Of note, PP2 has been shown to downregulate Akt in MM cells (36). This may explain the synergistic effects of sequential inhibition of the FAK/PI3-K/Akt pathway by LFA878 and each of the four tested Src family kinase inhibitors. In addition, more downstream inhibition, namely PI3-K inhibition by LY294002, provides similar results.

In conclusion, this is the first report on the LFA-1/ FAK/PI3-K/Akt survival pathway in MM and on the induction of apoptosis by inhibition of this pathway. Further studies are urgently needed to learn more about LFA-1 signalling in primary MM cells, constitutive LFA-1 survival pathway activation in the different TC (translocation/cyclin D) sub-groups, and its significance in in vivo models. Finally, sub-clones need to be addressed since MM subpopulations with high LFA-1 expression seem to consist of particular myelogenic (8), aggressive proliferating (21), and drug resistant $(3,5)$ cells.

\section{Acknowledgements}

This study was supported by the Fellowship 2005/28 awarded by the European Hematology Association (to R.S.).

\section{References}

1. Hideshima T, Chauhan D, Podar K, Schlossman RL, Richardson $\mathrm{P}$ and Anderson $\mathrm{KC}$ : Novel therapies targeting the myeloma cell and its bone marrow microenvironment. Semin Oncol 28: 607-612, 2001.

2. Lokhorst HM, Lamme T, De Smet M, et al: Primary tumor cells of myeloma patients induce interleukin- 6 secretion in long-term bone marrow cultures. Blood 84: 2269-2277, 1994.

3. Schmidmaier R, Morsdorf K, Baumann P, Emmerich B and Meinhardt G: Evidence for cell adhesion mediated drug resistance of multiple myeloma cells in vivo. Int J Biol Markers 21: 218-222, 2006.

4. Silvestris F, Cafforio P, Calvani N and Dammacco F: Impaired osteoblastogenesis in myeloma bone disease: role of upregulated apoptosis by cytokines and malignant plasma cells. $\mathrm{Br} \mathrm{J}$ Haematol 126: 475-486, 2004

5. Schmidmaier R, Baumann P, Simsek M, Dayyani F, Emmerich B and Meinhardt G: The HMG-CoA reductase inhibitor simvastatin overcomes cell adhesion-mediated drug resistance in multiple myeloma by geranylgeranylation of Rho protein and activation of Rho kinase. Blood 104: 1825-1832, 2004.

6. Olson DL, Burkly LC, Leone DR, Dolinski BM and Lobb RR: Anti-alpha4 integrin monoclonal antibody inhibits multiple myeloma growth in a murine model. Mol Cancer Ther 4: 91-99, 2005 .

7. Ahsmann EJ, Benschop RJ, De Gruyl TD, Faber JA, Lokhorst HM and Bloem AC: A novel flow cytometric assay for the quantification of adhesion of subsets within a heterogeneous cell population; analysis of lymphocyte function-associated antigen-1 (LFA-1)-mediated binding of bone marrow-derived primary tumour cells of patients with multiple myeloma. Clin Exp Immunol 93: 456-463, 2003.

8. Asosingh K, Vankerkhove V, van Riet I, van Camp B and Vanderkerken K: Selective in vivo growth of lymphocyte function-associated antigen-1-positive murine myeloma cells. Involvement of function-associated antigen-1-mediated homotypic cell-cell adhesion. Exp Hematol 31: 48-55, 2003.

9. Schmidmaier R, Baumann P and Meinhardt G: Cell-cell contact mediated signalling - no fear of contact. Exp Oncol 28: 12-15, 2006.

10. Gauvreau GM, Becker AB, Boulet LP, et al: The effects of an anti-CD11a mAb, efalizumab, on allergen-induced airway responses and airway inflammation in subjects with atopic asthma. J Allergy Clin Immunol 112: 331-338, 2003.

11. Papp K, Bissonnette R, Krueger JG, et al: The treatment of moderate to severe psoriasis with a new anti-CD11a monoclonal antibody. J Am Acad Dermatol 45: 665-674, 2001.

12. Hourmant M, Bedrossian J, Durand D, et al: A randomized multicenter trial comparing leukocyte function-associated antigen-1 monoclonal antibody with rabbit antithymocyte globulin as induction treatment in first kidney transplantations. Transplantation 62: 1565-1570, 1996.

13. Weitz-Schmidt G, Welzenbach K, Dawson J and Kallen J: Improved lymphocyte function-associated antigen-1 (LFA-1) inhibition by statin derivatives: molecular basis determined by X-ray analysis and monitoring of LFA-1 conformational changes in vitro and ex vivo. J Biol Chem 279: 46764-46771, 2004.

14. Schmidmaier R, Simsek M, Baumann P, Emmerich B and Meinhardt G: Synergistic antimyeloma effects of zoledronate and simvastatin. Anticancer Drugs 17: 621-629, 2006.

15. Meinhardt G, Dayyani F, Jahrsdorfer B, Baumgart J, Emmerich B and Schmidmaier R: Treosulfan is an effective inducer of cell death in myeloma cell lines and primary myeloma cells from patients. Br J Haematol 122: 892-899, 2003. 
16. Kawano MM, Huang N, Tanaka H, et al: Homotypic cell aggregations of human myeloma cells with ICAM-1 and LFA-1 molecules. Br J Haematol 79: 583-588, 1991.

17. Vermeulen K, van Bockstaele DR and Berneman ZN: The cell cycle: a review of regulation, deregulation and therapeutic targets in cancer. Cell Prolif 36: 131-149, 2003.

18. Mohamed AJ, Nore BF, Christensson B and Smith CI: Signalling of Bruton's tyrosine kinase, Btk. Scand J Immunol 49: 113-118, 1999.

19. Tatsumi T, Shimazaki C, Goto H, et al: Expression of adhesion molecules on myeloma cells. Jpn J Cancer Res 87: 837-842, 1996.

20. Vacca A, Di Loreto M, Ribatti D, et al: Bone marrow of patients with active multiple myeloma: angiogenesis and plasma cell adhesion molecules LFA-1, VLA-4, LAM-1, and CD44. Am J Hematol 50: 9-14, 1995.

21. Robillard N, Pellat-Deceunynck C and Bataille R: Phenotypic characterization of the human myeloma cell growth fraction. Blood 105: 4845-4848, 2005.

22. Alexandrakis MG, Passam FH, Sfiridaki K, et al: Clinical significance of circulating endothelial adhesion molecules (sE-selectin and sICAM) in untreated multiple myeloma patients. Clin Chim Acta 349: 39-43, 2004

23. Richardson P, Schlossman R, Jagannath S, et al: Thalidomide for patients with relapsed multiple myeloma after high-dose chemotherapy and stem cell transplantation: results of an openlabel multicenter phase 2 study of efficacy, toxicity, and biological activity. Mayo Clin Proc 79: 875-882, 2004.

24. Scudla V, Budikova M, Bacovsky J, Opichalova D and Farbiakova V: Relation of serum levels of the soluble cytoadhesion molecules SVCAM-1 and sICAM-1 to selected factors in the cytokine network in multiple myeloma. Cas Lek Cesk 139: 401-406, 2000.

25. Dubertret L, Sterry W, Bos JD, et al: Clinical experience acquired with the efalizumab (Raptiva) (CLEAR) trial in patients with moderate-to-severe plaque psoriasis: results from a phase III international randomized, placebo-controlled trial. $\mathrm{Br} J$ Dermatol 155: 170-181, 2006.

26. Hideshima T, Bergsagel PL, Kuehl WM and Anderson KC: Advances in biology of multiple myeloma: clinical applications. Blood 104: 607-618, 2004.
27. Sanchez-Martin L, Sanchez-Sanchez N, Gutierrez-Lopez MD, et al: Signaling through the leukocyte integrin LFA-1 in T cells induces a transient activation of Rac-1 that is regulated by Vav and PI3K/Akt-1. J Biol Chem 279: 16194-16205, 2004.

28. Bataille R, Jego G, Robillard N, et al: The phenotype of normal, reactive and malignant plasma cells. Identification of "many and multiple myelomas' and of new targets for myeloma therapy. Haematologica 91: 1234-1240, 2006.

29. Tabassam FH, Umehara H, Huang JY, et al: Beta2-integrin, LFA-1, and TCR/CD3 synergistically induce tyrosine phosphorylation of focal adhesion kinase [pp125(FAK)] in PHAactivated T cells. Cell Immunol 193: 179-184, 1999.

30. Tai YT, Podar K, Catley L, et al: Insulin-like growth factor-1 induces adhesion and migration in human multiple myeloma cells via activation of beta1-integrin and phosphatidylinositol 3'-kinase/AKT signaling. Cancer Res 63: 5850-5858, 2003.

31. Qiang YW, Yao L, Tosato G and Rudikoff S: Insulin-like growth factor I induces migration and invasion of human multiple myeloma cells. Blood 103: 301-308, 2004.

32. Glenney JR Jr and Zokas L: Novel tyrosine kinase substrates from Rous sarcoma virus-transformed cells are present in the membrane skeleton. J Cell Biol 108: 2401-2408, 1989.

33. Mitra SK and Schlaepfer DD: Integrin-regulated FAK-Src signaling in normal and cancer cells. Curr Opin Cell Biol 18: 516-523, 2006.

34. Ishikawa $\mathrm{H}$, Tsuyama $\mathrm{N}$ and Kawano MM: Interleukin-6induced proliferation of human myeloma cells associated with CD45 molecules. Int J Hematol 78: 95-105, 2003.

35. Mahmoud MS, Ishikawa H, Fujii R and Kawano MM: Induction of CD45 expression and proliferation in U-266 myeloma cell line by interleukin-6. Blood 92: 3887-3897, 1998.

36. Podar K, Mostoslavsky G, Sattler M, et al: Critical role for hematopoietic cell kinase (Hck)-mediated phosphorylation of Gab1 and Gab2 docking proteins in interleukin 6-induced proliferation and survival of multiple myeloma cells. J Biol Chem 279: 21658-21665, 2004. 